\title{
First Confirmed Cases of 2019 Novel Coronavirus in a University Hospital in Turkey: Housemate Internists
}

Oğuz Abdullah Uyaroğlu', [MD]

ORCID: 0000-0003-0440-2026

Nursel Çalık Başaran', [MD]

ORCID: 0000-0002-1290-6905

Lale Özışık', [MD]

ORCID: 0000-0002-3494-8997

Zahit Taş², [MD]

ORCID: 0000-0003-1292-5951

Gülçin Telli Dizman², [MD]

ORCID: 0000-0001-8195-3345

Ahmet Çağkan İnkaya², [MD]

ORCID: 0000-0001-7943-8715

Şehnaz Alp², [MD]

ORCID: 0000-0001-9424-8346

Mine Durusu Tanrı̈över', [MD]

ORCID: 0000-0001-9565-4389

Gökhan Metan², [MD]

ORCID: 0000-0002-2676-4557

Gülay Sain Güven², [MD]

ORCID: 0000-0001-8176-9767

'Hacettepe University Faculty of Medicine, Department of Internal Medicine, Section of General Internal Medicine, Ankara, Turkey

${ }^{2}$ Hacettepe University Faculty of Medicine, Department of Infectious Diseases and Clinical Microbiology,

Ankara, Turkey

Corresponding Author: Oğuz Abdullah Uyaroğlu Hacettepe University Faculty of Medicine, Department of Internal Medicine, Division of General Internal Medicine 06100 Sıhhıye, Ankara, Turkey.

E-mail: oguzuyaroglu@hotmail.com

https://doi.org/10.32552/2020.ActaMedica.476

\section{w ABSTRACT Con}

Objective: On 30 January 2020, WHO declared that coronavirus disease 2019 (COVID-19) outbreak has met the criteria for a Public Health Emergency of International Concern. Here, we report the clinical features and outcome of the first two cases of COVID-19 in a university hospital in Turkey, who were house-mate internists.

Case Presentation: Our first patient was a 28-year-old female who works as a resident in the Internal Medicine Department of a 1200-bed, tertiary care University Hospital. Her initial symptom was fatigue. She developed dry cough, nausea, and myalgia. She had no fever, no shortness of breath, or any other complaints. As the 2019-nCoV PCR test was positive, oral hydroxychloroquine and azithromycin were commenced. After the positive PCR test of the first patient, we screened 85 subjects who had unprotect-ed exposure to her; most of them were her colleagues and other healthcare workers. Test results of all but one were negative. The only person with a positive test result was the 35-year-old female who was the housemate and also a colleague of the first patient.

Discussion: These two cases showed us the desperate situation of COVID-19. To fight an infectious disease that had a high transmission rate, it is important to the infection chain between source and vulnerable subject and transmission route. Unfortunately, to find all infected sources is not possible every time, due to asymptomatic or mild cases, or with low sensitivity diagnostic tests. So, we need to do vigorous isolation pre-cautions and to test more people to end up this pandemic.

Keywords: COVID-19, 2019-nCoV, transmission, healthcare workers, internists 


\section{INTRODUCTION}

The World Health Organization (WHO) China Country Office was informed about accumulated pneumonia cases of unknown etiology in Wuhan City, Hubei Province of China on 31 December 2019 [1]. The Chinese authorities isolated and identified a new type of coronavirus, named 2019 novel coronavirus (2019-nCoV, then SARS-CoV-2). The dis-ease spread rapidly to many countries all over the world and WHO declared COVID-19 as a global pan-demic. The first case identified in Turkey was report-ed by the Ministry of Health on March 11, 2020. As of June 10, 2020, over 150.000 laboratoryconfirmed cases and 4,500 total deaths had been recorded in Turkey [2].

Here, we report the clinical features and outcome of the first two cases of COVID-19, who were housemate internists.

\section{CASE PRESENTATIONS}

\section{Case 1}

Our first patient was a 28-year-old female, who has been working as a resident in the Internal Medicine Department. Her initial symptom was fatigue. After the initial symptom, she developed dry cough, nausea and myalgia. She visited the Infectious Diseases Outpatient Clinic after her complaints did not disappear within 3 days. She had no travel history or no contact with anyone who had a travel history or a confirmed diagnosis of COVID-19. She had never been in contact with a known COVID-19 patient before. The physical examination was normal and the vital signs were within normal range (See Table 1). Chest radiography was normal. Laboratory tests revealed leukopenia and mild thrombocytopenia. Other test results are shown in Table 2. A nasopharyngeal swab specimen was obtained and sent for detection of 2019-nCoV by real time reverse transcriptase polymerase chain reaction (PCR). As the PCR test turned out to be positive, she was hospitalized and isolated in a single room, with contact and droplet precautions. After the onset of illness, her cough, nausea and myalgia worsened. She developed fever in follow-up. Her respiratory rate was still 18 breaths/minute. She had no chest discomfort and no need for oxygen support. Blood and urine cultures, respiratory tract bacterial and viral multiplex PCR were negative. The chest computerized tomography $(\mathrm{CT})$ revealed bilaterally peripheral ground glass opacities with irregular borders, especially in basal areas of right lung. Findings were compatible with COVID-19 pneumonia (Figure 1). After checking the QTc interval (390 msec), the patient was put on hydroxychloroquine $(400 \mathrm{mg}$ b.i.d on day one, then $200 \mathrm{mg}$ b.i.d), and azithromycin (500 mg on day one, then $250 \mathrm{mg} /$ day for 4 days) treatment. During her follow-up, her complaints regressed except for nausea, which got worse and was thought to be related to azithromycin and hydroxychloroquine. Intravenous ondansetron was started as symptomatic treatment. She developed loss of taste on the following. On the 4th day of treatment, she was free of fever, nausea and taste loss. After 5 days of treatment, the patient was discharged home with the advice of completing the isolation period at home for 14 days.

On March 22, 2020, after the positive PCR test of the first patient, we screened hospital staff who had un-protected exposure to her $(\mathrm{N}=85)$; most of them be-ing her colleagues and other healthcare workers. Test results of all but one were negative. Eighty-four people with negative test results were informed about COVID-19 and were recommended to use sur-gical masks inside the hospital, and they were rec-ommended to refer for a second test in case symp-toms suggestive of COVID-19 develop.

\section{Case 2}

The only person with a positive test result among 85 staff was the 35-year-old female who was the housemate and also colleague of the first patient.

She had only dry cough and fatigue for the last 3 days (See Table 1). She had no significant past medical history. She was also hospitalized and isolated in a single room with contact and droplet precautions. The physical examination and the vital signs on the day of admission were normal. Chest radiography showed prominent bronchovascular branches and localized reticular opacities in the lower zones of the lungs bilaterally. Chest CT revealed two peri-fissural millimetric nodules on the right lung were, which were interpreted as non-specific for COVID-19 pneu-monia (Figure 2). D-dimer was significantly elevat-ed. Other test results are listed in Table 2 . She also developed fever on the 2 nd day of admission. 
Table 1. Symptoms and maximum body temperatures with regards to the day of illness and the day of hospitalization

\begin{tabular}{|c|c|c|c|c|c|c|c|c|}
\hline & \multicolumn{3}{|c|}{ Before Admission } & \multicolumn{5}{|c|}{ During the Hospitalization } \\
\hline & March 19 & March 20 & March 21 & March 22 & March 23 & March 24 & March 25 & March 26 \\
\hline \multicolumn{9}{|l|}{ CASE 1 Symptoms } \\
\hline $\begin{array}{l}\text { Body temperature, }{ }^{\circ} \mathrm{C} \\
\text { (maximum) }\end{array}$ & - & - & - & 36.5 & 38.2 & 38.5 & 37.0 & 36.5 \\
\hline Cough & - & + & + & + & + & + & - & - \\
\hline Dyspnea & - & - & - & - & - & - & - & - \\
\hline Sputum & - & - & - & - & - & - & - & - \\
\hline Sore throat & - & - & - & - & - & - & - & - \\
\hline Rhinorrhea & - & - & - & - & - & - & - & - \\
\hline Fatigue & + & + & + & + & + & + & + & + \\
\hline Myalgia & - & + & + & + & + & + & + & + \\
\hline Nausea & - & + & + & + & + & + & - & - \\
\hline Vomiting & - & - & - & - & - & - & - & - \\
\hline Diarrhea & - & - & - & - & - & - & - & - \\
\hline Abdominal Discomfort & - & - & - & - & - & - & - & - \\
\hline Loss of smell & - & - & - & - & - & - & - & - \\
\hline Loss of taste & - & - & - & - & + & + & - & - \\
\hline \multicolumn{9}{|l|}{ CASE 2 Symptoms } \\
\hline $\begin{array}{l}\text { Body temperature, }{ }^{\circ} \mathrm{C} \\
\text { (maximum) }\end{array}$ & - & - & - & 36.2 & 38.5 & 37.3 & 36.4 & 36.5 \\
\hline Cough & - & + & + & + & + & + & - & - \\
\hline Dyspnea & - & - & - & - & - & - & - & - \\
\hline Sputum & - & - & - & - & - & - & - & - \\
\hline Sore throat & - & - & - & - & - & - & - & - \\
\hline Rhinorrhea & - & - & - & - & - & - & - & - \\
\hline Fatigue & + & + & + & + & + & + & + & + \\
\hline Myalgia & - & - & - & + & + & + & + & + \\
\hline Nausea & - & - & - & - & + & - & - & - \\
\hline Vomiting & - & - & - & - & - & - & - & - \\
\hline Diarrhea & - & - & - & - & - & - & - & - \\
\hline Abdominal Discomfort & - & - & - & - & + & + & - & - \\
\hline Loss of smell & - & - & - & - & + & + & + & - \\
\hline Loss of taste & - & - & - & - & - & - & - & - \\
\hline
\end{tabular}

Blood and urine cultures, respiratory tract bacterial and viral multiplex PCR were negative. The same treat-ment protocol was initiated. On the 3th day of treat-ment, her fever resolved, and cough reduced. She developed abdominal discomfort, nausea and min-imal loss of smell. This patient was also discharged after 5 days of treatment with the advice of com-pleting the isolation period at home for 14 days.

\section{DISCUSSION}

Health care workers (HCWs) on the front line who are directly involved in the examination, diagnosis, treatment, and care of patients with COVID-19, are at high risk of developing the disease. In the first re-ports; out of 44,672 confirmed COVID-19 cases, $1716(3,8 \%)$ were HCWs. Overall, $14.8 \%$ of confirmed cas-es among HCWs were classified as severe or 
Table 2. Laboratory findings of the cases

\begin{tabular}{|c|c|c|c|}
\hline Laboratory Parameters & Reference Range & Case 1 & Case 2 \\
\hline Hemoglobin (g/dL) & $11.7-15.5$ & 13.5 & 13.7 \\
\hline Hematocrit (\%) & $34.5-46.3$ & 40.3 & 40.2 \\
\hline White-cell count $(\mu \mathrm{L})$ & $4.100-10.200$ & 2.500 & 7.900 \\
\hline Absolute neutrophil count ( $\mu \mathrm{L})$ & $1.800-6.400$ & 1.020 & 5.190 \\
\hline Absolute lymphocyte count ( $\mu \mathrm{L})$ & $1200-3600$ & 1.210 & 1.680 \\
\hline Absolute monocyte count $(\mu \mathrm{L})$ & $300-900$ & 270 & 690 \\
\hline Absolute eosinophil count ( $\mu \mathrm{L})$ & $100-500$ & 30 & 220 \\
\hline Absolute basophil count $(\mu \mathrm{L})$ & $0-200$ & 10 & 80 \\
\hline Platelet count ( $\mu \mathrm{L})$ & $159.000-388.000$ & 145.000 & 220.000 \\
\hline Sodium (mEq/L) & $136-146$ & 135 & 136 \\
\hline Potassium (mEq/L) & $3.5-5.1$ & 3.68 & 3.63 \\
\hline Chloride (mEq/L) & $101-109$ & 100 & 105 \\
\hline Blood urea nitrogen (mg/dL) & $6-20$ & 16.4 & 13.8 \\
\hline Creatinine (mg/dL) & $0.51-0.95$ & 0.69 & 0.65 \\
\hline Alanine aminotransferase (U/L) & $<35$ & 10 & 27 \\
\hline Aspartate aminotransferase (U/L) & $<35$ & 21 & 20 \\
\hline Creatine kinase (U/L) & $<145$ & 75 & 71 \\
\hline Lactate dehydrogenase (U/L) & $<247$ & 152 & 154 \\
\hline CK-MB $(\mu \mathrm{g} / \mathrm{L})$ & $0.6-6.3$ & 0.6 & 0.4 \\
\hline Myoglobin ( $\mu \mathrm{g} / \mathrm{L})$ & $14.3-65.8$ & 22.3 & 24.8 \\
\hline Troponin (ng/L) & $8.4-18.3$ & 2.8 & $<2.3$ \\
\hline Internalized normalized ratio & $0.8-1.2$ & 1.16 & 0.97 \\
\hline Fibrinogen (mg/dL) & $180-350$ & 250.09 & 375.48 \\
\hline D-dimer (mg/L) & $0-0.55$ & 0.32 & $\begin{array}{l}0.74 \text { (Day 1) } \\
1.18 \text { (Day 2) }\end{array}$ \\
\hline C-reactive protein (mg/dL) & $0-0.8$ & 0.402 & 0.274 \\
\hline Procalcitonin (ng/mL) & $0-0.1$ & 0.03 & 0.03 \\
\hline Erythrocyte sedimentation rate ( $\mathrm{mm} /$ hour) & $0-25$ & 13 & 17 \\
\hline Ferritin $(\mu \mathrm{g} / \mathrm{L})$ & $11-307$ & 20.5 & 44.1 \\
\hline
\end{tabular}

critical, and 5 deaths were observed [3]. Recently; an esti-mated $3000 \mathrm{HCW}$ s have been infected and at least 22 have died in China. Transmission to family mem-bers of HCWs is widely reported [4]. HCWs' infection is also widespread throughout Europe. The Health Ministry of Spain reported that out of 40,000 con-firmed COVID-19 cases, 5,400 (nearly $14 \%$ ) were medical professionals. It is stated that in Italy, France and Spain, more than 30 health care professionals have died due to COVID-19 and thousands of others have had to self-isolate [5].

After the positive PCR test of the first patient, we screened 85 subjects who had suspicious exposure with her; most of them were her colleagues and other health care workers. We thought that knowledge of the status of 2019-nCov infection among HCWs would provide important insights for the participating of our hospital regarding the infection status of staff and would inform hospital policies on testing algorithms for our personnel and on infection prevention measures.

But the infection source or index case for our cases could not be determined. In our cases, the PCR results of subjects who had suspicious exposure with the first patient in the workplace were negative, but only the person who had house contact was positive. This can be explained by importance of higher viral load, and longer exposure time and closer contact are important for viral transmission. But on the other hand, lower sensitivity and specificity of the laboratory methods, real history of close contact, inappropriate specimen collection, can lead false 

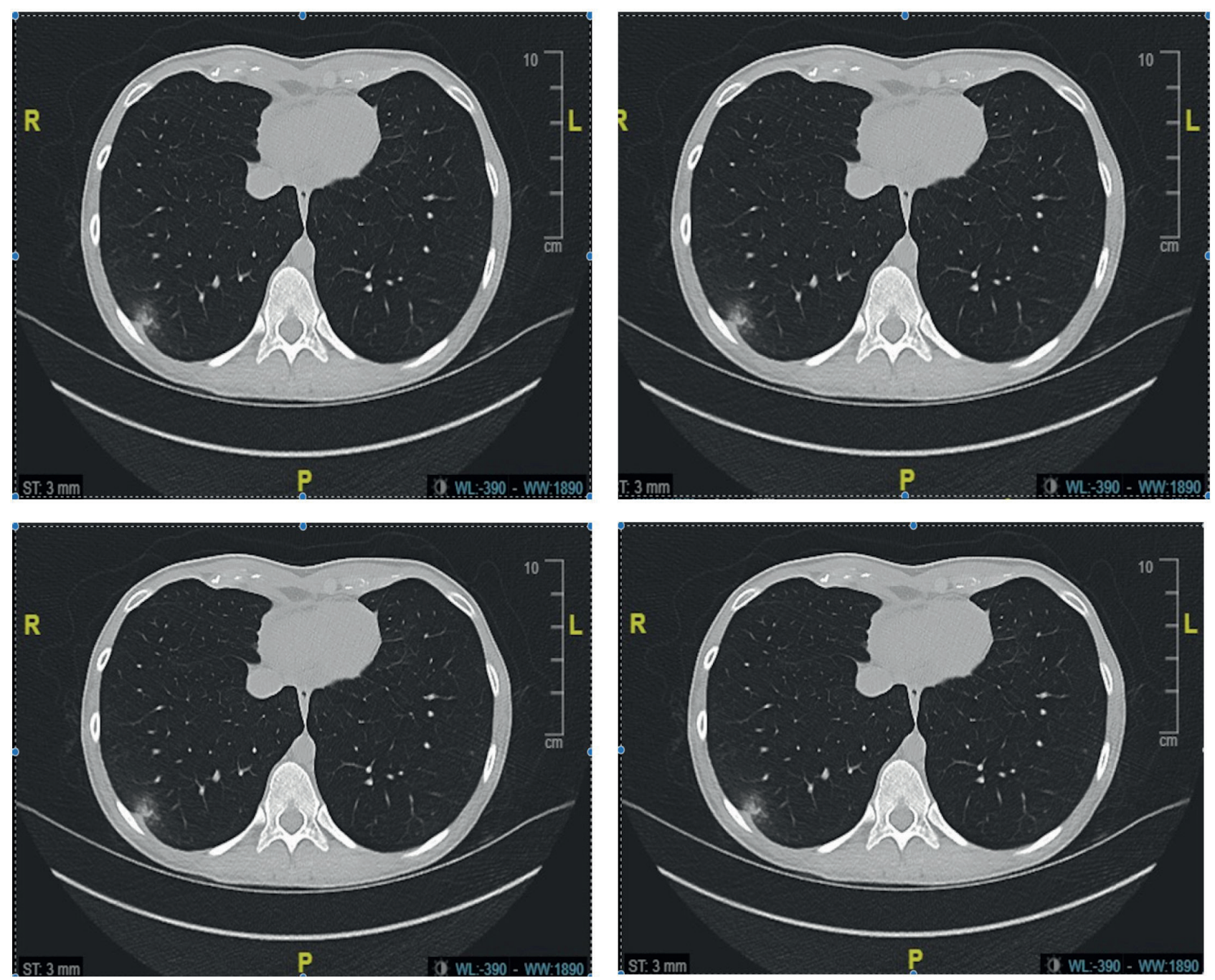

Figure 1. Chest Computerized Tomography of Patient 1: Bilaterally, peripheral ground glass opacities with irregular borders which are prevalent in the right basal zone. Findings are compatible with COVID-19 pneumonia.


Figure 2. Chest Computerized Tomography of Patient 2: Two peri-fissural millimetric nodules on the right. Insignificant findings in terms of COVID 19 pneumonia. 
negative test results. Our patients were not working in the same ward. Before the cases were detected, the suspected patients in the internal medicine wards had been evaluated for COVID-19, and there were no positive test results or lung $C T$ positive for COVID-19. So it was difficult to determine if these cases were nosocomial, or community acquired. As previously reported in the literature [6], these two cases have shown that considerable transmission is occurring among close contacts.

In a study from Netherlands, HCWs with mild respiratory complaints and without epidemiological link (contact with confirmed case or visited areas with active circulation) were tested for 2019-nCov. 1,097 HCWs in nine hospitals were tested; 45 (4.1\%) were positive. The results suggest unnoticed community transmission, with a potential risk of nosocomi-al transmission [7].

These two cases should remind us, HCWs, the importance of standart, and whenever indicates special infection control precautions. Although in the absence of any known infectious disease, health institutions are under higher risk of contagious disease risk for both HCWs, and also for the patients. These two cases showed us the desperate situation about COVID-19. To fight an infectious disease which had high transmission rate, it is important to brake the infection chain between source and vulnera-ble subject and transmission route. Unfortunately, detecting all infected sources is not possible every time, due to asymptomatic or mild cases, or with low sensitivity diagnostic tests. So, we need to do vigorous isolation precautions, and to test more people to end up this pandemic.

Regarding the patients' clinics, the first patient described loss of smell, while the second patient described loss of taste. Citing a growing number of cases around the globe, the American Academy of
Otorhynolaryngology - Head and Neck Surgery and Ear-Nose-Throat (ENT) - UK each issued warnings about patients who tested positive for 2019-nCoV with the only symptom being a lost or altered sense of smell or taste [8]. There is growing publication that sudden loss of smell and taste is an early stimulant in the course of COVID-19 $[9,10]$. In a cross sec-tional study from Italy, found that 20 of 59 patients (ages 50-74) interviewed, reported a smell or taste loss [11]. Currently, there have been reports of taste and smell disorders related to COVID-19 from multi-ple countries around the world and our case reports can contribute to the literature in this regard.

Our report of the first confirmed two cases of 2019$\mathrm{nCoV}$ in our hospital illustrates several aspects of this emerging pandemic, including our treatment approach to patients, the course of symptoms and an example in relation to loss of smell and taste, which is increasingly being reported.

\section{ACKNOWLEDGEMENT}

N/A

\section{COMPLIANCE WITH ETHICAL STANDARDS}

The authors declare no potential conflicts of interest.

\section{FUNDING}

None of the authors received a financial support and/or specific funding for this work.

*Written consent has taken from our patients. 


\section{ne) REFERENCES CQm}

[1] WHO. Novel coronavirus - China. Jan 12, 2020. Avaible from: http://www.who. int/csr/don/12-january-2020-novel-coronavirus-china/en/ (accessed Jan 19, 2020)

[2] T.C Sağlık Bakanlığı. Türkiye'deki güncel durum. Avaible from: https://covid19.saglik.gov.tr/

[3] Wu Z, McGoogan JM. Characteristics of and Important Lessons From the Coronavirus Disease 2019 (COVID-19) Outbreak in China: Summary of a Report of 72314 Cases From the Chinese Center for Disease Control and Prevention. JAMA. 2020;323(13):1239-1242.

[4] Adams JG, Walls RM. Supporting the Health Care Workforce During the COVID-19 Global Epidemic. JAMA.2020;323(15):1439-1440.

[5] Raphael Minder, Elian Peltier.Virus Knocks Thousands of Health Workers Out of Action in Europe. Avaible from: https://nyti.ms/3dob8BT

[6] Bi, Qifang, et al. "Epidemiology and transmission of COVID-19 in 391 cases and 1286 of their close contacts in Shenzhen, China: a retrospective cohort study."The Lancet Infectious Diseases(2020).
[7] Reusken CB, Buiting A, Bleeker-Rovers C, et al. Rapid as-sessment of regional SARS-CoV-2 community transmission through a convenience sample of healthcare workers, the Netherlands, March 2020. Euro Surveill. 2020;25(12):2000334.

[8] AAO-HNS: Anosmia, Hyposmia, and Dysgeusia sytmpoms of Coronavirus Disease. Available from: https://www.entnet.org/content/aao-hns-anosmia-hyposmia-and-dysgeusia-symptoms-coronavirus-disease

[9] Ling Mao, Mengdie Wang, Shanghai Chen, et al. Neurological Manifestations of Hospitalized Patients with COVID-19 in Wuhan, China: a retrospective case series study. medRxiv 2020.02.22.20026500.

[10] David Brann, Tatsuya Tsukahara, Caleb Weinreb, et al. Non-neural expression of SARS-CoV-2 entry genes in the olfactory epithelium suggests mechanisms underlying anosmia in COVID-19 patients. bioRxiv 2020.03.25.009084.

[11] Andrea Giacomelli, Laura Pezzati, Federico Conti, et al. Self-reported olfactory and taste disorders in SARSCoV-2 patients: a cross-sectional study, Clinical Infectious Diseases, , ciaa330. 